\title{
P2P computing for intelligence of things
}

\section{Sunmoon Jo ${ }^{1}$. Jieun Lee ${ }^{2}$ Jungsoo $\mathrm{Han}^{3}$ - Supratip Ghose ${ }^{4}$}

Published online: 21 February 2020

(C) Springer Science+Business Media, LLC, part of Springer Nature 2020
With the development of the Internet of Things (IoT) and machine to machine (M2M), the hyper-connected peer-topeer (P2P) networking age has come, and all objects related to human life are able to connect through the Internet. Through deep learning, human-machine interaction, and convergence in various areas, many different types of objects are connected in an Internet-based network environment. Many objects used in human life send and receive data in real time through a diversity of network technologies including RFID, NFC, WIFI, Bluetooth, ZigBee, GPS, and 4G. In particular, with the introduction of $5 \mathrm{G}$, more access to massive amounts of content makes it possible to provide diverse services. New IoT devices using intelligence computing continue to be developed and distributed. As the types and numbers of IoT devices have increased, networks have gradually become more complex. For an efficient use of a network, there have been more demands and studies of P2P computing technology. Numerous IoT devices are used to

Sunmoon Jo

sunmoon.joh@gmail.com

Jieun Lee

jieun.lee.0072@gmail.com

Jungsoo Han

jungsoo.han.k@gmail.com

Supratip Ghose

sgresearch@gmail.com

1 Deptartment of Computer Information Technology Education, Paichai University, Doma 2-dong, Seo-gu, Daejeon, Republic of Korea

2 Korea Convergence Society, 65, Buldang 21-ro, Seobuk-gu, Cheonan-si, Chungcheongnam-do, South Korea

3 Division of Information \& Communication, Baekseok University, 76, Munam-ro, Anseo-dong, Dongnam-gu, Cheonan-si, Chungcheongnam-do, South Korea

4 Deptartment of Computer Science and Engineering, University of Information Technology \& Sciences, Dhaka, Bangladesh establish small or large networks through $\mathrm{P} 2 \mathrm{P}$ computing. The convergence of diverse technologies, such as IoT, P2P, ambient intelligence, data mining, machine learning, deep learning, and distributed computing, facilitates the development of convergence industries including smart cities, smart farms, and smart health. At the center of smart industries is IoT, which makes it possible to connect objects. For an efficient processing, a P2P network needs to be researched. P2P computing is an intelligence technology that positively influences people's lifestyles and helps create a newly added-value and improve the quality of human life.

It is aimed at sharing the vision, research outcomes, and solutions to convergence technologies, such as P2P applications, built-in P2P, intelligent P2P networks, and P2P-based distributed processing between researchers and developers in IoT-based smart industries. Through cooperation among research and development teams, P2P technologies are applied to establish an environment for solving problems that arise during the use of IoT devices, networks, and data.

The first paper by Kim et al. [1], P2P computing for a trusted network of personalized IoT services is proposed. P2P computing focuses on the protection of IoT service users and data. To identify a reliable device, familiarity scores are evaluated through the experience of a particular exchange and a connection with a data message. At a gateway, the masks generated during an exchange and message transmission are compared. Based on the comparative results, familiarity scores are added. In this way, it is possible to create P2P networking with high reliability. The paper by Cho et al. [2], the selective switching of double-transmission schema in a multi-LED hybrid VLC-P2P network is suggested. VCL is good at establishing an indoor light infrastructure with the use of a P2P network. However, indirect light and the wavelength of multiple light sources causes interference, which lowers the network performance. To minimize the interference, the researchers proposed a double-transmission with selective switching. The proposed method divides areas of interference into three types depending on the received power, and separates and recovers the received signals through an 
interference cancellation technique in regions with relatively weak interference, and then sends them to the cell boundary region. In this way, it minimizes any interference, but still incurs complexity in the system.

The paper by Lee et al. [3], the authors propose a blockchain-based item reputation management for a customized manufacturing service in a P2P system. This is an item reputation management technique that focuses on higher reliability and accuracy of the IoT-based manufacturing system. By identifying malicious reviewers and excluding their feedback, it is possible to classify and predict the decision-making of real customers In terms of the reputation of the current item, the researchers exclude reviews from malicious users by not considering the mean of the reputation scores of a product made by the same manufacturer or the reputation of relevant items. In particular, by excluding the values of the users who continuously give a low evaluation score to other products made by the same manufacturer, the proposed method processes malicious feedback. The paper by Noor Alleema et al. [4], a method using volunteer nodes of ant colony optimization (VNACO) routing is proposed to minimize the delay time of P2P MANET. A mobile ad hoc network (MANET) is not dependent on the sub structure necessary to establish a wireless network with mobility. Nodes are often disconnected owing to their mobility. Researchers have solved this problem through the use of VNACO. If a relay node is out of communication range, a volunteer node sends dropped data to the relay node.

The paper by Uma Maheswari et al. [5], repetitive node categorization based on stable clustering and energy-efficient $\mathrm{P} 2 \mathrm{P}$ communication in a wireless sensor network is introduced. With an increase in the number of application programs, notorious activities are also increased through unreliable sensor nodes, consumer a limited amount of sensor power and thereby influencing the overall performance. Researchers have separated unreliable sensors through the use of a reliable topology recognition and setting step, a grouping step, and a data collection, tree structure, and relaxation step. In this manner, energy is managed more efficiently. The paper by Park et al. [6], a mobile IoT device summary method is proposed using the unique features of a P2P web search engine and content. Because it is easy to find the unique structure of the information using potential features and the k-mean algorithm, a query is optimized. It is possible to automatically determine a proper sentence that fits the minimized query without a user intervention. A summary is executed in the server, and more tasks can be performed given the user's limited device resources. The paper by Hong [7], a P2P networking-based IoT sensor node proof using a block chain is proposed. M2M has a variety of senor nodes and is vulnerable to jamming and tampering attacks. A sink node uses a block chain to execute a simple hash function and to provide network integrity, non-repudiation, and safe certification. The paper by Park et al. [8], an open health cloud for P2P-based drug management system is proposed. The developed system is designed to provide a smooth virtual cloud service through the cloud established through pre-reserved communication and M2M. The proposed system utilizes a chatbot-type mobile health service to prevent drug abuse in everyday life.

The paper by Mun et al. [9], an authentication method based on the arm movement in a P2P-based M2M is proposed. The proposed method utilizes a wearable IoT device in a $\mathrm{P} 2 \mathrm{P}$ system to measure the direction of movement, analyze EMG signals, and identify users. From the signals collected by an IoT sensor, a potential feature vector is extracted using a wavelet noise removal algorithm. In the extracted feature vector, a multi-class-SVM is applied for classification and identification. The paper by Priya et al. [10], an optimized road method of a hybrid P2P cloud data center using a resource minimization algorithm and efficient road optimization is proposed. For an efficient road optimization in M2M, game theory is applied. The developed scheduling algorithm, supporting parallel processing with the use of a two-tier VM, is used for dynamic load balancing. A heuristic-based multi-load scheduling method is used to reduce resource usage and response time.

The paper by Yoo et al. [11], an edge computing model using a deep neural network through P2P networking is proposed. The proposed method has a combination of data parallel processing and model parallel processing to solve the communication overhead for the sharing of distributed data. To improve the response speed of deep learning, an efficient parallel processing using a $\mathrm{P} 2 \mathrm{P}$ network and edge computing model is applied. A module is created with multiple edge nodes, and transactions are generated in a module and connected to a distributed DNN model. To reduce the computing power of a server model, a modularized deep neural network that processes big data in parallel is established. The paper by Kim et al. [12], a hybrid P2P networking distribution web crawler using Amazon Web Services (AWS) for Smart Work News BigData is established. A crawler generates a lot of traffic in a web server and is thus similar to a DDoS attack. For this reason, a web server tries to block a crawler. To solve this problem, the researchers used the cloud service platform offered by AWS. It is possible to define a crawler client's role. Even if a particular node is blocked, the entire system works normally. The crawler is not blocked because it accesses the web server for one client at a time. The paper by Park et al. [13], a multi-robot mapping system that selects the P2P communication protocol from a given mapping or flower plan was developed to solve the mapping problem and map split problem. Based on a centralized overlay network, this is a scalable P2P network in which a base station broadcasts a control command to all robots. With the use of a P2P network, it is possible to share a mapping and path plan between robots, reduce the number of operations, and solve the problem of limited resources.

This fine collection of articles was achieved by fruitful collaborations. We would like to thank Prof. Xuemin S. 
Shen, the Editor-in-Chief of Peer-to-Peer Networking and Applications for his great efforts and support throughout the publication.

\section{References}

1. Kim DY, Lee A, Kim S (2019) P2P computing for trusted networking of personalized IoT services. Peer-to-Peer Networking and Applications:1-9. https://doi.org/10.1007/s12083-019-00737-z

2. Han D, Cho M (2019) Selective switching dual-transmission scheme in multi-LED hybrid VLC-P2P networking system. Peerto-Peer Networking and Applications 11:1166-1175. https://doi. org/10.1007/s12083-017-0606-0

3. Lee YJ, Lee KM, Lee SH (2019) Blockchain-based reputation management for custom manufacturing service in the peer-to-peer networking environment. Peer-to-Peer Networking and Applications: 1-13. https://doi.org/10.1007/s12083-019-00730-6

4. Noor Alleema N, Siva Kumar D (2019) Volunteer nodes of ant colony optimization routing for minimizing delay in peer to peer MANETs. Peer-to-Peer Networking and Applications:1-11. https:// doi.org/10.1007/s12083-019-00772-w

5. Uma Maheswari P, Ganeshbabu TR (2019) Repetitive node categorization technique based reliable clustering and energy efficient communication in P2P wireless sensor network. Peer-to-Peer Networking and Applications. https://doi.org/10.1007/s12083019-00768-6

6. Park S, Cha BR, Chung K, Kim JW (2019) Mobile IoT device summarizer using P2P web search engine and inherent characteristic of contents. Peer-to-Peer Networking and Applications:1-10. https://doi.org/10.1007/s12083-019-00780-w

7. Hong S (2019) P2P networking based internet of things (IoT) sensor node authentication by Blockchain. Peer-to-Peer Networking and Applications:1-11. https://doi.org/10.1007/s12083-01900739-x

8. Chung K, Park RC (2019) P2P-based open health cloud for medicine management. Peer-to-Peer Networking and Applications:113. https://doi.org/10.1007/s12083-019-00791-7

9. Shin J, Islam MR, Rhaim MA, Mun HJ (2019) Arm movement activity based user authentication in P2P systems. Peer-to-Peer Networking and Applications. https://doi.org/10.1007/s12083019-00775-7

10. Priya B, Gnanasekaran T (2019) To optimize load of hybrid P2P cloud data-center using efficient load optimization and resource minimization algorithm. Peer-to-Peer Networking and Applications:1-12. https://doi.org/10.1007/s12083-019-00795-3

11. Chung K, Yoo H (2019) Edge computing health model using P2Pbased deep neural networks. Peer-to-Peer Networking and Applications:1-10. https://doi.org/10.1007/s12083-019-00738-y

12. Kim YY, Kim YK, Kim DS, Kim MH (2019) Implementation of hybrid P2P networking distributed web crawler using AWS for smart work news big data. Peer-to-Peer Networking and Applications:1-12. https://doi.org/10.1007/s12083-019-00841-0

13. Tien M, Park YY, Jung KH, Kim SY, Kye JE (2019) Performance evaluation on the accuracy of the semantic map of an autonomous robot equipped with P2P communication module. Peer-to-Peer Networking and Applications:1-13. https://doi.org/10.1007/ s12083-019-00851-y

Publisher's note Springer Nature remains neutral with regard to jurisdictional claims in published maps and institutional affiliations.

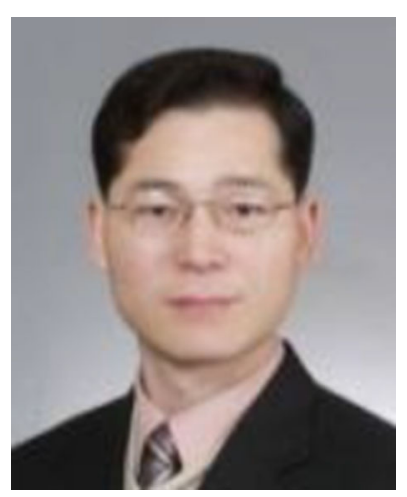

SunMoon Jo is a Professor in the Department of Computer Information Technology Education, Paichai University, South Korea. His research interests include telecommunication, XML, security, knowledge system, deep learning, hybrid P2P, and wireless communication. He serves as Director and a member of the Editorial Committee of the Korea Knowledge Information Technology Society, as Director of the Korea Contents Association, as Member of the Korean Society for Internet Information, as Member of the Korea Information Processing Society, as Member of the Institute of Electrical and Electronics Engineers, as Steering Committees of International Conference on Digital Policy \& Management.

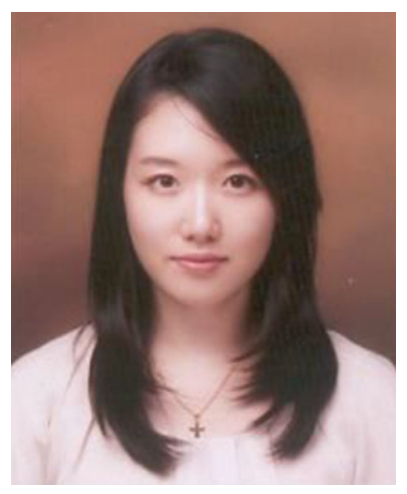

Jieun Lee is the Manager of "The Korea convergence Society", Republic of Korea. She managed conferences including International Conference Convergence Technolo gy, International Conference on Internet of Thing and Convergence and International Research Confe rence on Innovation, Technology and Sustainability. Her research topics are Sensibility Design, IT Con vergence, Digital Computing, Bigdata Mining, Hybrid Networking Systems, Hybrid P2P Protocols, Graphics, and Digital Content. Furthermore, she has controlled special issues of prestigious international journals. Also, she is editorial board members of several International Joumals.

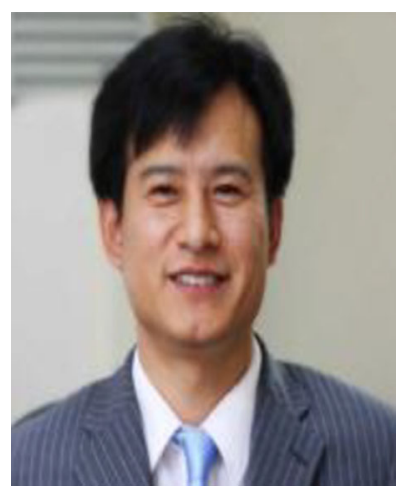

JungSoo Han is a Professor in the Division of Information \& Commu nication, Baekseok University, Cheonan City, Chungnam, South Korea. In 2014, he researched Convergence IT and Creative Education Methodology at California State University Fullerton as an Exchange Professor. His research topics include data mining, contents planning, telemedicine, knowledgebased decision support systems, intelligent systems, convergence P2P, HCI, hybrid $\mathrm{P} 2 \mathrm{P}$, and telecommunication systems. He has edited books on computer science and convergence technology. He serves as Executive Editing Director of the International Conference on Convergence Content (ICCC), as General CoChair of the International Conference on Digital Policy \& Management (ICPDM), as General Co-Chair for steering committees of the International Conference on Convergence Technology (ICCT), as Vice President of the Korea convergence society, as Vice President and a member of the Editorial Committee of the Society of Digital Policy \& Management. 


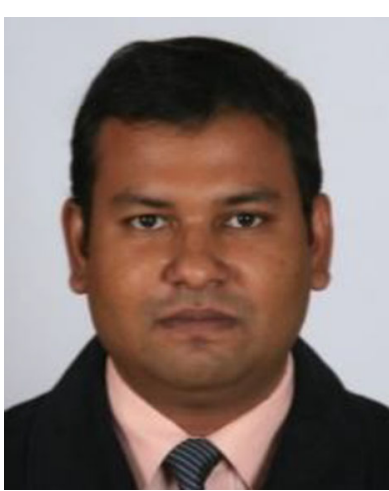

Supratip Ghose graduated from the Department of Applied Physics and Electronics, University of Rajshahi, with a BSc and an MSc in 1995 and 1998, respectively, and received his $\mathrm{PhD}$ in Information Engineering, from the Department of Computer Science \& Information Engineering, Inha University, South Korea, in 2007. Dr. Ghose began his academic career as an Adjunct Lecturer at Inha University. After obtaining his $\mathrm{PhD}$, he served as an Assistant Professor in the Department of Computer Science and Engineering in UIC-BNU-HKBU in Zhuhai, China, the University of Saint Joseph, Macau, China, and Brac University in Dhaka, Bangladesh. He is currently serving as an Associate Professor in the Department of Computer Science and Engineering, University of Information Technology \& Sciences, Dhaka, Bangladesh. His research interests include Data Mining, Collaborative Filtering, Sentiment Analysis in Social Webs, IR frameworks, and Activity Recognition in Smartphones. 